\title{
Application Research on Motor Fault Monitoring on Audio Signal Recognition Technology with SOPC
}

\author{
GongQin $^{1, a}$, JunZhou $^{2, b}$
}

\author{
${ }^{1}$ School of Phisics and Engineering, Jianghan University, Wuhan 430056, Hubei,China; \\ ${ }^{2}$ School of Phisics and Engineering, Jianghan University, Wuhan 430056, Hubei,China; \\ aEmail: 497839993@qq.com, ${ }^{\mathrm{b}}$ Email: 867602524@qq.com
}

\begin{abstract}
Keywords: SOPC; STFT; Audio signal recognition; revolving speed; motor fault; online mon itoring

Abstract: This application research adopts the development kit named DE1-SoC, which can realize real-time collection about the voice signal of the motor rotation, and remove noises with anti aliasing filter. We research the corresponding spectrum in frequency domain after the combining STFT transformation. Using the corresponding algorithm, we can find the frequency spectrum component related to the speed, build relations between the frequency and the corresponding motor speed. Thus, the state information of motor running is obtained. Taking advantage of pattern recognition technology, the real-time running state of motor was compared with the spectrum diagram of the normal running, we can detect the workings of motor, and automatically the system identifies the failure status of motor in the running process.
\end{abstract}

\section{Introduction}

In the modern industrial production, motors are applied in various places. How to ensure the safety and normal running of the motor is a significant topic of the modern equipment operation maintenance and management. The methods of monitoring motor running state and fault diagnosis are various ${ }^{[1]}$.Among these, the noise monitoring and diagnosis method that can be realized on-line monitoring and fault diagnosis while not affecting the motor running condition is most convenient and effective because the audio signal of motor rotational noise contains abundant motor running state information and can be picked up easily. The Cyclone V SoC of Altera, integrated a RISC processor system to the FPGA as a complementary, not only has powerful parallel signal processing ability and can realize the complex algorithm flexibly, and also has a very low power consumption.DE1-SoC development kit shows great advantage in the motor fault detection with the audio signal recognition technology.

\section{Audio recognition algorithm and feature extraction}

\subsection{The combined short-time Fourier transform (STFT)}

When abnormal motor malfunctions occurs, its corresponding vibration or noise signal is non-stationary characteristics while the signal frequency components will change over time ${ }^{[2]}$. The corresponding signal processing uses time-frequency analysis method, including the Wiger-Ville distribution, STFT, the Gabor expansions, Wavelet transform and etc. The STFT is simple with high processing rate, but it has some defects in the treatment of non-stationary signal ${ }^{[3]}$.Using the improved STFT, it will be able to meet the requirements of motor fault signal analysis.

Time-varying signal $x(t)$ is defined as:

$$
\operatorname{STFT}_{x}(t, f)=\int_{-\infty}^{+\infty} x\left(t^{\prime}\right) g^{*}\left(t^{\prime}-t\right) e^{-j 2 \pi f t^{\prime}} d t^{\prime}
$$

Where $g^{*}\left(t^{\prime}-t\right)$ is conjugate of the sliding window $g\left(t^{\prime}-t\right)$, $t$ is the center of the window. STFT can be understood as the local spectrum of signal $x\left(t^{\prime}\right)$, while inhibiting signal outside the sliding window.

$X(f)$ and $G(f)$ is the Fourier transform of the signal $x(t)$ and the window function $g(t)$ 
respectively, STFT expression in the frequency domain is ${ }^{[3]}$ :

$$
\operatorname{STFT}_{x}(t, f)=e^{-j 2 \pi f t} \int_{-\infty}^{+\infty} X\left(f^{\prime}\right) G^{*}\left(f^{\prime}-f\right) e^{-j 2 \pi f t^{\prime}} d f^{\prime}
$$

In this way, the short-time Fourier transform has the time domain and frequency domain localization function simultaneously. It can realize local analysis of the signal time and frequency.

Time domain function $g(t)$ and frequency domain function $G(f)$ of the sliding window was determined respectively in the time domain and frequency domain signal interception. To determine the analysis precision of the Fourier transform, the narrower the window width, the higher the corresponding time resolution is. However, according to the uncertainty principle, a sliding window cannot has a narrow window width and narrow frequency window width at the same time. And short time Fourier transform has nothing to do with the frequency, the window size remains the same, it does not meet the request in the practical problem that the high frequency signal of time resolution should be higher than low frequency signal and vice versa.

Aiming at this limitation of STFT, two solutions are given. First, during the motor start-up phase, we research the problem with a long window function and reduce window length adaptively according to the increase of rotational speed, until a stable working condition. In the process of adaptive window function, it can be easily acquired the corresponding parameters which is relate to the motor speed. In the process of implementation, we also define the scope of adaptive window length which ensure the convergence of adaptive change. Second, we take advantage of the characteristics of the FPGA which is suitable for parallel computing, we can operate STFT which has different window lengths at the same time. This system uses three different window lengths to study at the same time, including window length relate to the rotational speed, twice its window length, the $1 / 2$ window length, which adapt to the fault pattern recognition in the process of motor running. Although motor operation and failure belongs to the non-stationary process, but due to the limit such as mechanical inertia factors which lead to the parameters such as its highest speed and speed change are relatively low, the above scheme can meet requirement about real-time and accuracy simultaneously completely. It is also easy to be implemented on FPGA to solve the limitation of the fixed window length of STFT transform.

\subsection{Motor speed relationship with feature frequency points}

Low frequency components of motor noise and its speed have a strong correlation. In general, motor electromagnetic noise frequency follows ${ }^{[4]}$ :

$$
f=\frac{k z n}{60}
$$

Among them, $n$ is the motor speed, $z$ is the motor structure parameter, and $k$ is the order.

By tracking the system, we take samples about running condition change of motor during the experiment that motor speed continuously improves. We get more groups of data and make FFT transform to obtain the spectrum parameters, the FFT transform at this time is correspond to short time Fourier transform. Through the analysis of the spectrum data, we find out the formant associated with speed change and follow up. When we find spectrum peak corresponds to the frequency doubled, the window size will be adjusted automatically. We can find out the frequency spectrum peak for the following tracking. Through the use of Matlab software we obtain the relation between the rotational speed and the feature frequency points that is basically a linear relationship, it conforms to the description of the formula (3), as shown in Fig 1. 


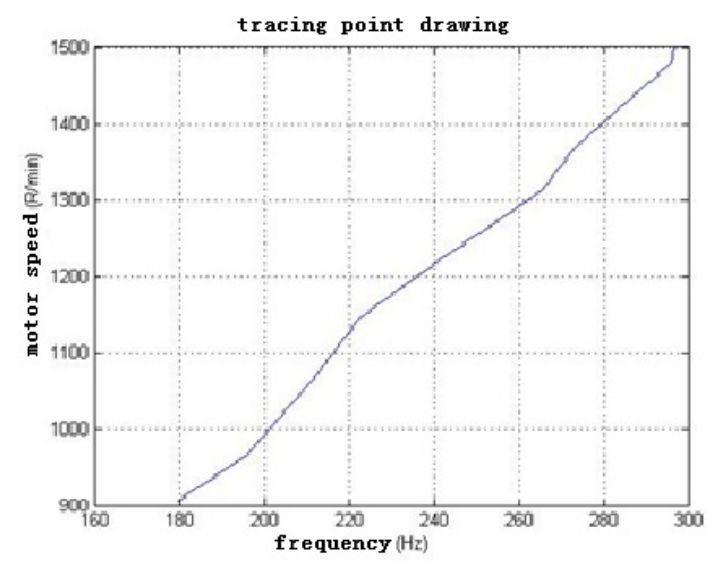

Fig 1 Motor speed change with abscissa spectrum peaks diagram

Through the MATLAB software in the ployfit() function on the fitting, a = polyfit (x, $\mathrm{f}$, 3 ), we can get three times polynomial fitting function :

$$
f=0.000110 x^{3}-0.0809 x^{2}+24.4271 x-1523.9349
$$

It proves that the speed we extracted from the frequency component is true.

\subsection{Motor fault relationship with audio noise of motor}

In the process of motor operation, failure will be reflected in the motor running in the audio signal to different types or different degree of fault. There are many differences between the audio signal spectrums. For example, if there is a loose connection, it can produce a strong vibration of 2 times of the feature frequency, as well as the sideband of a third of the feature frequency accompanied. If there is a rotor-bearing fault, it will appear two to four times of the feature frequency. Therefore, through the spectrum analysis we can locate the fault parts. If we combined it with its amplitude, sideband and amplitude modulation signal of high frequency band, we can also judge the degree of the fault ${ }^{[5][6]}$.

Through real-time detection of the spectrum in the process of motor running, comparing with the audio signal spectrum of the normal operation and studying the certain frequency components and their amplitudes, we can judge the fault type and fault degree. With pattern recognition technology, we can perform the intelligent judgment on the motor abnormal working state.

\section{System design based on the SOPC}

\subsection{System Design}

The system uses Altera DE1-SoC development kit. The DE1-SoC development kit presents a robust hardware design kit with Altera System-on-Chip (SoC) FPGA, which combines the latest dual-core Cortex-A9 embedded cores with industry-leading programmable logic for ultimate design flexibility. Users can now leverage the power of tremendous re-configurability paired with a high-performance, low-power processor system. SoC of Altera integrates an ARM-based hard processor system (HPS) consisting of processor, peripherals and memory interfaces tied seamlessly with the FPGA fabric using a high-band width interconnect backbone. The DE1-SoC development board includes hardware such as high-speed DDR3 memory, video and audio capabilities, Ethernet networking, and so on ${ }^{[7]}$. The system is stable and reliable, which is very suitable for the audio signal processing of this system. System flow chart is shown in Fig2. 


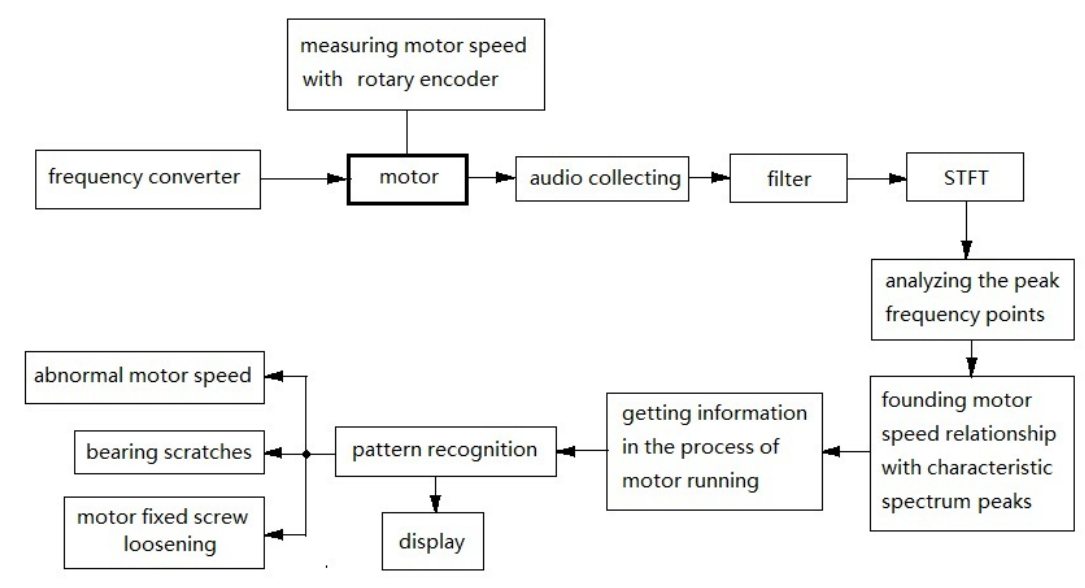

Fig2 system flowchart

We use the electret microphone to collect the motor operating audio signal and convert it to digital signal through the WM8731.Custom components we designed are added to the AVALON bus system as customized IP core. Afterwards, in hardcore of Cortex-A9, we operate directly on the acquisition module with a high-level language, and we realized hardware system which the ARM and FPGA resources can access each other.

Filtering out some irrelevant frequency signal by using anti-aliasing filter, we can reduce the amount of data processing.

Through research, we found that we can meet the requirements of the system for ordinary motor using an $8 \mathrm{KHz}$ sampling, 16 quantitative (about $90 \mathrm{~dB}$ dynamic range) of audio signal. As experimental study, with the 8192-point FFT transform (1 s), $1 \mathrm{~Hz}$ resolution can be achieved, and the corresponding 512 points $(62.5 \mathrm{~ms})$ can reach the resolution of the $16 \mathrm{~Hz}$.

By analyzing the audio signal spectrum of motor running noise, we find that there are multiple different peaks. They are not all correlated to the speed. Analyzing some peaks in the spectrum structure and recording the location of these peaks that is frequency, using the process that the motor speed gradually increase after the motor startup, we can get the position changes of the peaks and peaks that related to the motor speed. Tracking them, we can converse them to motor speed.

Through studying energy distribution of the motor operating frequency spectrum in the process of change, choosing appropriate length of FFT window, monitoring data and analyzing it according to the characteristics of various fault, we use pattern recognition techniques to compare the energy distribution with voice spectrum signal in the process of normal operation. As result we can learn whether the motor working state is normal and other possible related issues. In the Experiment, we have carried on three kinds of motor fault pattern recognition.

\subsection{Hardware design with FPGA}

We operate FFT transform by the MegaWizard in hardware implementation. Compared with the algorithm implementation with software, we can realize higher speed data processing, save more CPU resources, and take the good advantage of FPGA. The FPGA hardware design principle diagram is shown in Fig 3. 


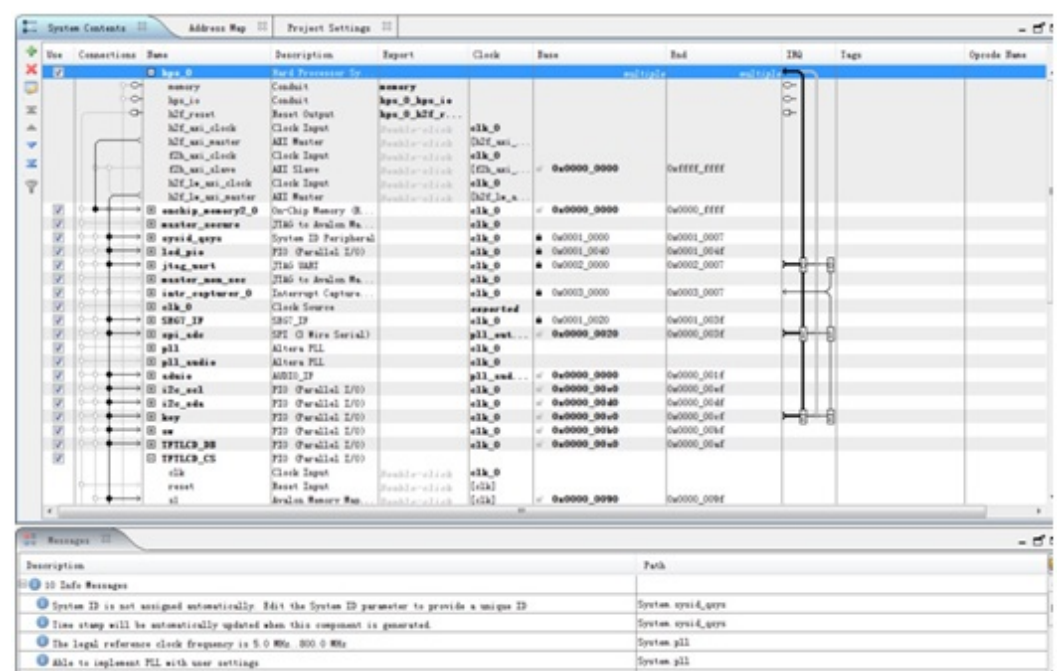

Fig3 the principle diagram of the hardware design with FPGA

FFT algorithm adopts high performance Fast Fourier Transform(FFT) processing IP core of Altera company. We realized high-speed complex FFT calculation with length of 512,1024,2048,4096, and 8192. We designed a top package file to bind the FFT IP core to the Avalon bus.

FFT operation data flow is controlled by the scatter - gather DMA. Altera scatter- gather DMA controller is DMA IP core based on the bus of Avalon- ST flow.

\section{Experimental analysis}

We use three-phase asynchronous motor for experience. The power of motor is $0.75 \mathrm{KW}$, connection method is $\mathrm{Y}$, working current is $1.88 \mathrm{~A}$, and rotate speed is $1390 \mathrm{R} / \mathrm{min}$. The test result of motor speed is showed in Table 1.

Table 1 Three-phase asynchronous motor speed test results and deviation

\begin{tabular}{|c|c|c|c|}
\hline $\begin{array}{c}\text { actual motor speed } \\
(\mathrm{R} / \mathrm{min})\end{array}$ & $\begin{array}{c}\text { measurement of motor } \\
\text { speed }(\mathrm{R} / \mathrm{min})\end{array}$ & $\begin{array}{c}\text { deviation value } \\
\text { (absolute value) }\end{array}$ & $\begin{array}{l}\text { measurement } \\
\text { accuracy }\end{array}$ \\
\hline 1500 & 1515 & 15 & $1.00 \%$ \\
\hline 1432 & 1433 & 1 & $0.07 \%$ \\
\hline 1396 & 1392 & 4 & $0.27 \%$ \\
\hline 1263 & 1277 & 14 & $1.10 \%$ \\
\hline 1135 & 1151 & 16 & $1.41 \%$ \\
\hline 1051 & 1056 & 5 & $0.48 \%$ \\
\hline 967 & 978 & 11 & $0.11 \%$ \\
\hline 886 & 887 & 1 & $1.41 \%$ \\
\hline
\end{tabular}

Learned from table 1, the maximum deviation of the rotational speed is $1.41 \%$, and the minimum deviation is $0.07 \%$, the average deviation is $0.70 \%$. The main factors that causing errors are the influence of environmental noise, the influence of frequency resolution and the influence of quantization error.

Under the condition of motor function, we collect the motor rotation voice and store the running state information such as speed, spectrum distribution and energy distribution. These data is stored in the memory of the DE1-SoC as the reference standard of fault detection. Through the experiment, it can prompt the abnormal of the motor speed when the speed exceed or under the normal speed signal in a certain amount. When the system detects the corresponding frequency peak point or harmonic point energy increasing, it indicates that a cyclical phenomenon such as scratches with the motor in the process of running. By testing the total energy change of the corresponding spectrum segment, such as the low frequencies, it hints that in the motor some fixed screws are loosening or fuselage vibration fault phenomenon is occurring at that moment. 


\section{Conclusions}

Taking the advantage of FPGA that has fast speed and strong anti-jamming capability on FFT algorithm, through the use of high performance Fast Fourier Transform(FFT) processing IP core, we realize a combined short-time Fourier transform (STFT) with different window lengths in hardware in parallel. With the adaptive window length according to the motor characteristics, we can meet the request on both the frequency and time resolution in motor running fault monitoring. On the base of system we designed, we use the pattern recognition techniques to detect the motor running fault. Experiments achieved a good effect on data processing accuracy and real-time monitoring performance.

\section{References}

[1] Wang Huizhong. Study on fault diagnosis technologies of motor. Industry and Mine Automation, 2015, 41(1): 40-44

[2]Chen Jin. Signal processing applications in machinery fault diagnosis. Journal of Vibration and Shock, 1999, 18(4): 84-87

[3]Xiao Ying, Feng Changjian. Combination Window Function of Short-time Fourier Transform Domain Representation. Journal of Detection \& Control, 2010, 32(3): 43-47

[4]Wei Yili. Speed Research and Exploration Based on Audio Method. electroacoustic technology, 2007, 31(B06): 4-7

[5]Lang Jianxun. Motor Rotor Fault Detection Method Based on Contrast Audio Technology. Journal of hubei institute for nationalities, 2013, 31(3):320-322

[6] Wang Qing-nian. Motor Noise Source Identification Based On Spectrum Analysis. Technical Acoustics,2009,28(4):528-531

[7] Information on

https://www.altera.com.cn/products/soc/portfolio/cyclone-v-soc/overview.html 